\title{
SCIENTIFIC REPRTS \\ Crystal structure of Pelagibacterium halotolerans PE8: New insight into its substrate-binding pattern
}

Received: 9 August 2016

Accepted: 17 May 2017

Published online: 30 June 2017
Ying-Yi Huo ${ }^{1}$, Suhua $\mathrm{Li}^{2}$, Jing Huang ${ }^{2}$, Zhen Rong ${ }^{1}$, Zhao Wang ${ }^{3}$, Zhengyang Li ${ }^{2}$, Rui $\mathrm{Ji}^{2}$, Siyun Kuang ${ }^{2}$, Heng-Lin Cui ${ }^{3}$, Jixi Li ${ }^{2} \&$ Xue-Wei Xu${ }^{1}$

Lysophospholipase_carboxylesterase (LPCE) has highly conserved homologs in many diverse species ranging from bacteria to humans, as well as substantial biological significance and potential therapeutic implications. However, its biological function and catalytic mechanism remain minimally investigated because of the lack of structural information. Here, we report the crystal structure of a bacterial esterase PE8 belonging to the LPCE family. The crystal structure of PE8 was solved with a high resolution of $1.66 \AA$. Compared with other homologs in the family, significant differences were observed in the amino acid sequence, three-dimensional structure, and substrate-binding pattern. Residue Arg79 undergoes configuration switching when binding to the substrate and forms a unique wall, leading to a relatively closed cavity in the substrate-binding pocket compared with the relatively more open and longer clefts in other homologs. Moreover, the mutant Met122Ala showed much stronger substrate affinity and higher catalytic efficiency because less steric repulsion acted on the substrates. Taken together, these results showed that, in PE8, Arg79 and Met122 play important roles in substrate binding and the binding pocket shaping, respectively. Our study provides new insight into the catalytic mechanism of $L P C E$, which may facilitate the development of structure-based therapeutics and other biocatalytic applications.

Esterases have important physiological roles and biotechnological applications because they can catalyze the hydrolysis of short-chain ester-containing molecules and produce carboxylates and alcohols ${ }^{1-3}$. Esterases belong to the lysophospholipase_carboxylesterase family (the LPCE family) ${ }^{4}$ and were previously classified in bacterial family VI by Arpigny and Jaeger ${ }^{1}$. This family includes the smallest carboxylesterase $(23-26 \mathrm{kDa})$ found to date, and bacterial carboxylesterases show high sequence similaritiy with their eukaryotic counterparts $(\sim 40 \%)^{1}$. LPCE family proteins play significant roles in human diseases. For example, human putative G $\alpha$-regulatory protein acyl thioesterase (APT1) has been well characterized as a modulator in the Ras signaling pathway and has been confirmed as a target for cancer therapeutics ${ }^{5,6}$. Human lysophospholipase-like 1 (LYPLAL1) might be a triacylglycerol lipase involved in obesity ${ }^{7,8}$. In addition, a bacterial carboxylesterase (FTT258) from Francisella tularensis, a causative agent of tularemia ${ }^{9,10}$, has been investigated as a novel drug target ${ }^{11}$. Overall, LPCE family enzymes are increasingly pharmaceutically interesting as potential therapeutic targets. Nevertheless, our understanding of the LPCE family is very limited. Currently, the crystal structures of only six LPCE family proteins have been reported, including Rhodobacter sphaeroides $\mathrm{RspE}^{12}$, Pseudomonas aeruginosa $\mathrm{PA} 3859^{13}$, $P$. fluorescens esterase $\mathrm{II}^{14}$, F. tularensis $\mathrm{FTT}_{25}{ }^{11}$, human APT $1^{15}$ and human LYPLAL1 ${ }^{7}$.

The LPCE family member PE8 was recently identified by our group ${ }^{16,17}$. The biochemical characterization of PE8 revealed that it is an alkaline esterase and a potential industrial biocatalyst ${ }^{18}$. PE8 exhibits enantioselective hydrolysis of prochiral dimethyl 3-(4-fluorophenyl)glutarate (3-DFG), generating (R)-3-(4-fluorophenyl) glutarate $((R)-3-M F G)^{18}$, a precursor of important pharmaceutical compounds, such as the antidepressant $(-)$-paroxetine hydrochloride ${ }^{19}, 20$. In this study, we obtained and analyzed the crystal structure of PE8 to gain new insight into the catalytic mechanism of LPCE family enzymes.

${ }^{1}$ Key Laboratory of Marine Ecosystem and Biogeochemistry, Second Institute of Oceanography, State Oceanic Administration, Hangzhou, 310012, China. ${ }^{2}$ State Key Laboratory of Genetic Engineering, Collaborative Innovation Center of Genetics and Development, School of Life Sciences, Shanghai Engineering Research Center of Industrial Microorganisms, Fudan University, Shanghai, 200438, China. ${ }^{3}$ College of Food and Biological Engineering, Jiangsu University, Zhenjiang, 212013, China. Correspondence and requests for materials should be addressed to J.L. (email: lijixi@fudan.edu.cn) orX.-W.X. (email:xuxw@sio.org.cn) 
A

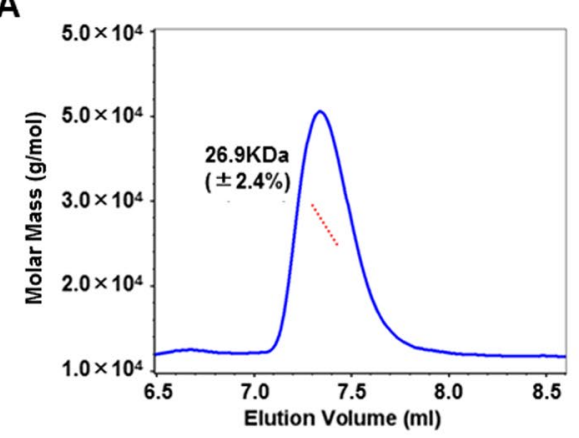

C

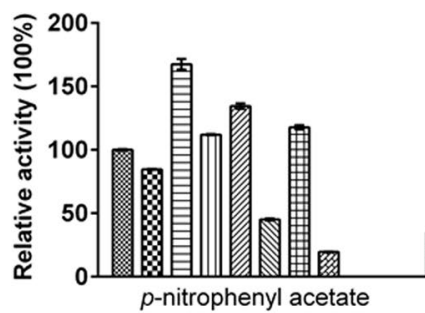

B

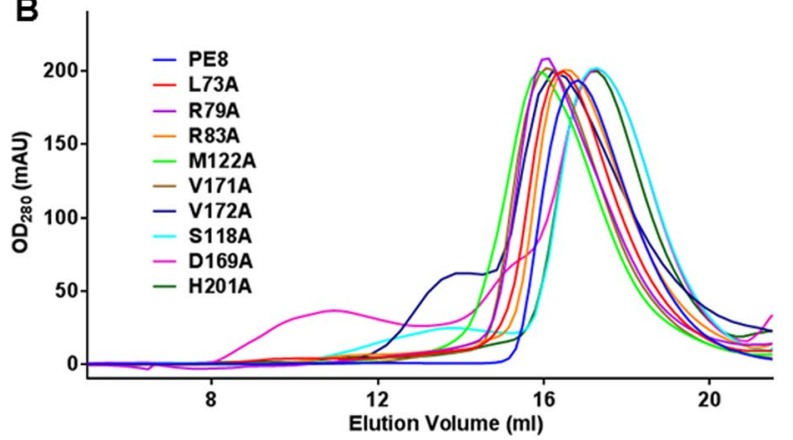

Figure 1. Gel filtration profile and enzymatic activities of PE8 and mutants. (A) MALS analysis of PE8. The MW of PE8 was $26.9 \mathrm{kDa}( \pm 2.4 \%)$, indicating that PE8 is a monomer in solution. (B) Gel filtration profiles of PE8 and mutants on a Superdex 200 10/300 column. Wild-type PE8 and its mutants formed monomers in solution. Mutants L73A, R79A, R83A, M122A, V171A and H201A changed slightly, whereas mutants V172A, S118A and D169A showed heterogeneity upon gel filtration. (C) The enzymatic activities of wild-type PE8 and its mutants were determined using the following substrates: $p$-NP acetate, $p$-NP butyrate, $p$-NP hexanoate and $p$-NP octanoate.

\section{Results and Discussion}

Overall structure. The diffracting dataset of the PE8 crystal was integrated into monoclinic space group $\mathrm{P} 2{ }_{1}$ with two molecules per asymmetric unit and a resolution of $1.66 \AA$. However, multi-angle light scattering (MALS) analysis showed that the molecular weight (MW) of PE8 was $26.9 \mathrm{kDa}( \pm 2.4 \%)$ (Fig. $1 \mathrm{~A})$, consistent with the theoretical MW of $6 \times$ His fusion PE8 $(25.4 \mathrm{kDa})$, and revealed that PE8 existed as a monomer in solution. Additionally, 329 water molecules, one polyethylene glycol (PEG) monomethyl ether (MME) 550 molecule and one glycerol molecule were modeled. The final refined model had an $R_{\text {work }}$ of $16.84 \%$ and an $R_{\text {free }}$ of $20.52 \%$. The crystallographic statistics for data collection and structure refinement are summarized in Table 1.

The molecular structure of PE8 had a typical $\alpha / \beta$-hydrolase fold ${ }^{21-23}$, containing seven predominantly parallel $\beta$ strands $(\beta 1, \beta 2$, and $\beta 5-\beta 9)$ surrounded by six $\alpha$ helices $(\alpha 1-\alpha 6)$ (Figs 2 and S1). The $\beta$-strands formed a parallel $\beta$-sheet in the order of $\beta 1$ (antiparallel to all the others), $\beta 3, \beta 2, \beta 6, \beta 7, \beta 8$ and $\beta 9$, with helices $\alpha 1$ and $\alpha 6$ on one side and $\alpha 2, \alpha 3, \alpha 4$ and $\alpha 5$ on the other side (Fig. 3A).

Compared with the known structures of other LPCE family members, the main differences were found in the short $\beta$-strands, helix $\alpha 4$, the short $\alpha$ helices and the $3_{10}$ helices (Supplementary Figure S1). Helix $\alpha 4$ connects $\beta 7$ with $\beta 8$ in PE8, R. sphaeroides RspE $^{12}$ and human LYPLAL1 ${ }^{7}$. However, in P. fluorescens esterase II ${ }^{14}$, P. aeruginosa PA3859 $9^{13}$ and human APT $1^{15}$, strands $\beta 7$ and $\beta 8$ are connected by long loops containing short $\alpha$ helices or $3_{10}$ helices. In addition, on the loop between strand $\beta 3$ and helix $\alpha 2$ (i.e., loop $\beta 3$ ), there are four short antiparallel $\beta$-strands $(\beta 4, \beta 5, \beta \mathrm{A}$ and $\beta \mathrm{B})$ in esterase II and APT1. In contrast, $\beta \mathrm{A}$ and $\beta \mathrm{B}$ of loop $\beta 3$ are replaced with a short helix in RspE or a winding loop in PE8, PA3859 and LYPLAL1 (Supplementary Figure S1).

The $\beta$-strands $\beta 6, \beta 8$ and $\beta 9$ provide the framework onto which the catalytic residues (Ser118, Asp169 and His201 in PE8) are placed (Figs 2 and 3A). The remaining $\beta$ strands and $\alpha$ helices are not directly involved in the formation of the catalytic site, and thus, the differences in the secondary structure mentioned above may not influence the catalysis of the active site directly.

Active site. Sequence analysis and the three-dimensional (3D) structure revealed that the catalytic triad residues of PE8 consist of Ser118, Asp169 and His201, which are located on the C-terminal sides of $\beta$ strands $\beta 6, \beta 8$ and $\beta 9$ in the central $\beta$ sheets (Figs 2 and 3 ) and are conserved in esterases ${ }^{1}$. The catalytic site is located on the loops outside of the $\alpha / \beta / \alpha$-sandwich structure, and no lid covers the catalytic site. The catalytic residue Ser 118 is located in the conserved GFSQG motif (Fig. 2). The hydrogen bond distance within the catalytic triad is $2.7 \AA$ from Ser118-O $\gamma$ to His201-Nع2 and $2.7 \AA$ from His201-N $\delta 1$ to Asp169-O $\delta 1$ (Fig. 3B). To confirm the roles of the three amino acid residues, site-directed mutagenesis was performed to replace these residues with alanine. The activities of mutant S118A against $p$-nitrophenyl ( $p$-NP) acetate, $p$-NP butyrate, $p$-NP hexanoate and $p$-NP octanoate were $19.5 \pm 0.2 \%, 16.3 \pm 0.5 \%, 1.3 \pm 0.2 \%$ and $0 \%$, respectively, compared with the wild-type enzyme (Fig. 1C). The replacement of catalytic residues Ser118 or Asp169 with alanine led to a complete loss of activity 


\begin{tabular}{|c|c|}
\hline Parameters & PE8 \\
\hline \multicolumn{2}{|l|}{ Diffraction data } \\
\hline Wavelength $(\AA)$ & 0.9785 \\
\hline Resolution range $(\AA)$ & $50.0-1.66(1.69-1.66)^{\mathrm{a}}$ \\
\hline Space group & $\mathrm{P} 2$ \\
\hline \multicolumn{2}{|l|}{ Unit cell } \\
\hline a, b, c $(\AA)$ & $41.772,73.398,66.403$ \\
\hline$\alpha, \beta, \gamma\left({ }^{\circ}\right)$ & $90.00,102.37,90.00$ \\
\hline Unique reflections & 46061 \\
\hline Completeness (\%) & $99.1(99.6)$ \\
\hline$R_{\text {merge }}(\%)^{\mathrm{b}}$ & $8.4(47.1)$ \\
\hline $\mathrm{I} / \sigma(\mathrm{I})$ & $22.5(3.17)$ \\
\hline Wilson B-factor & 21.42 \\
\hline \multicolumn{2}{|l|}{ Refinement statistics } \\
\hline Resolution range $(\AA)$ & $36.7-1.66(1.72-1.66)^{\mathrm{a}}$ \\
\hline$R_{\text {work }}{ }^{\mathrm{c}} / R_{\text {free }}(\%)^{\mathrm{d}}$ & $16.84 / 20.52$ \\
\hline No. atoms & 3580 \\
\hline No. residues & 438 \\
\hline No. PEG MME 550 & 1 \\
\hline No. glycerol & 1 \\
\hline No. water molecules & 329 \\
\hline \multicolumn{2}{|l|}{ RSMD } \\
\hline Bond lengths $(\AA)$ & 0.020 \\
\hline Bond angles $\left({ }^{\circ}\right)$ & 2.02 \\
\hline Average B-factor $\left(\AA^{2}\right)$ & 26.37 \\
\hline Ramachandran favored (\%) & 98 \\
\hline Ramachandran outliers (\%) & 0.46 \\
\hline PDB code & 5DWD \\
\hline
\end{tabular}

Table 1. Statistics from the data collection and refinement of PE8. ${ }^{a}$ Values in parentheses refer to data in the highest resolution shell. ${ }^{\mathrm{b}} R_{\text {merge }}=\sum\left|\mathrm{I}_{\mathrm{i}}-\langle\mathrm{I}\rangle\right| / \sum|\mathrm{I}|$, where $\mathrm{I}_{\mathrm{i}}$ is the intensity of an individual reflection and is the average intensity of that reflection. ${ }^{c} R_{\text {work }}=\sum|| \mathrm{F}_{\mathrm{o}}|-| \mathrm{F}_{\mathrm{c}}|| / \sum\left|\mathrm{F}_{\mathrm{o}}\right|$, where $\mathrm{F}_{\mathrm{o}}$ and $\mathrm{F}_{\mathrm{c}}$ are the observed and calculated structure factors of reflections, respectively. ${ }^{\mathrm{d}} R_{\text {free }}$ was calculated as $R_{\text {work }}$ using $5 \%$ of the reflections that were selected randomly and omitted from refinement.

against $p$-NP acetate, $p$-NP hexanoate and $p$-NP octanoate, with approximately only $5 \%$ of residual activity retained against $p$-NP butyrate (Fig. $1 \mathrm{C}$ ).

Compared with the known structures of LPCE family homologs, the oxyanion hole of PE8 is likely formed by nitrogen atoms of Tyr29 and Gln119, as observed for RspE (Tyr and Gln) but not for esterase II, PA3859, FTT258 and human APT1 (Leu and Gln) or human LYPLAL1 (Ser and Gln) (Figs 2 and 3B). In PE8, the oxyanion hole is occupied by a water molecule in each chain (data not shown). These water molecules might be candidates for the nucleophilic attack on the acylated enzyme ${ }^{13}$, followed by the release of the enzyme in its resting form.

Structural comparison of PE8 with other LPCE family homologs. A search for related models in the Protein Data Bank (PDB) using DALI ${ }^{24}$ yielded the best match with homologs belonging to the LPCE family, giving alignments of $197-212$ residues (22-46\% identity) with root-mean-square deviations (RSMD) of $2.4-1.7 \AA$ (Table 2). The homolog with the highest structural similarity and the highest sequence identity was R. sphaeroides RspE (PDB ID 4FHZ, Z score $=33.9, \mathrm{RMSD}=1.7 \AA ̊$ for $212 \mathrm{C} \alpha$ atoms, identity $=46 \%$, Table 2 ), followed by esterase II (PDB 1AUO), PA3859 (PDB 3CN9), APT1 (PDB 1FJ2), LYPLAL1 (PDB 3U0V) and FTT258 (PDB 4F21). Although the sequence identities between PE8 and the five homologs are relatively low, ranging from $22 \%$ to $26 \%$, these homologs still show high 3D structural similarities with low RSMD (2.1-1.7 $)$, mainly because of their relatively conserved $\alpha / \beta$-hydrolase fold, which contains a $\beta$-sheet with seven strands surrounded by five or six $\alpha$ helices (Fig. 4A).

Superimposing these homologs also revealed their similar features and overall folds. The core $\alpha / \beta$-hydrolase fold structure, including the catalytic triad and oxyanion hole, is highly conserved, especially in $\beta$ strands $\beta 6, \beta 8$ and $\beta 9$ and helices $\alpha 3, \alpha 5$ and $\alpha 6$ in the loops (Fig. 4A,C). High structural variability can be observed in helices $\alpha 1, \alpha 4$, and the $\beta 3$ loop (Fig. 4A), suggesting that these structures are not essential for the catalytic activity.

Among these regions, the $\beta 3$ loop shows the most significant variation in its amino acid sequence and secondary and 3D structures (Figs 2,4 and S1). The $\beta 3$ loop shows a higher B-factor within the PE8 structure (Fig. 4B), implying flexibility in its structure and function. Highly variable loops have also been described in LPCE family homologs previously ${ }^{11,15}$. This winding loop is believed to be responsible for the substrate specificity and conformational changes of these homologs ${ }^{13,25}$ and is observed to cause the open and closed conformations and affect the catalytic activity and membrane binding of F. tularensis FTT2 $258^{11}$. 

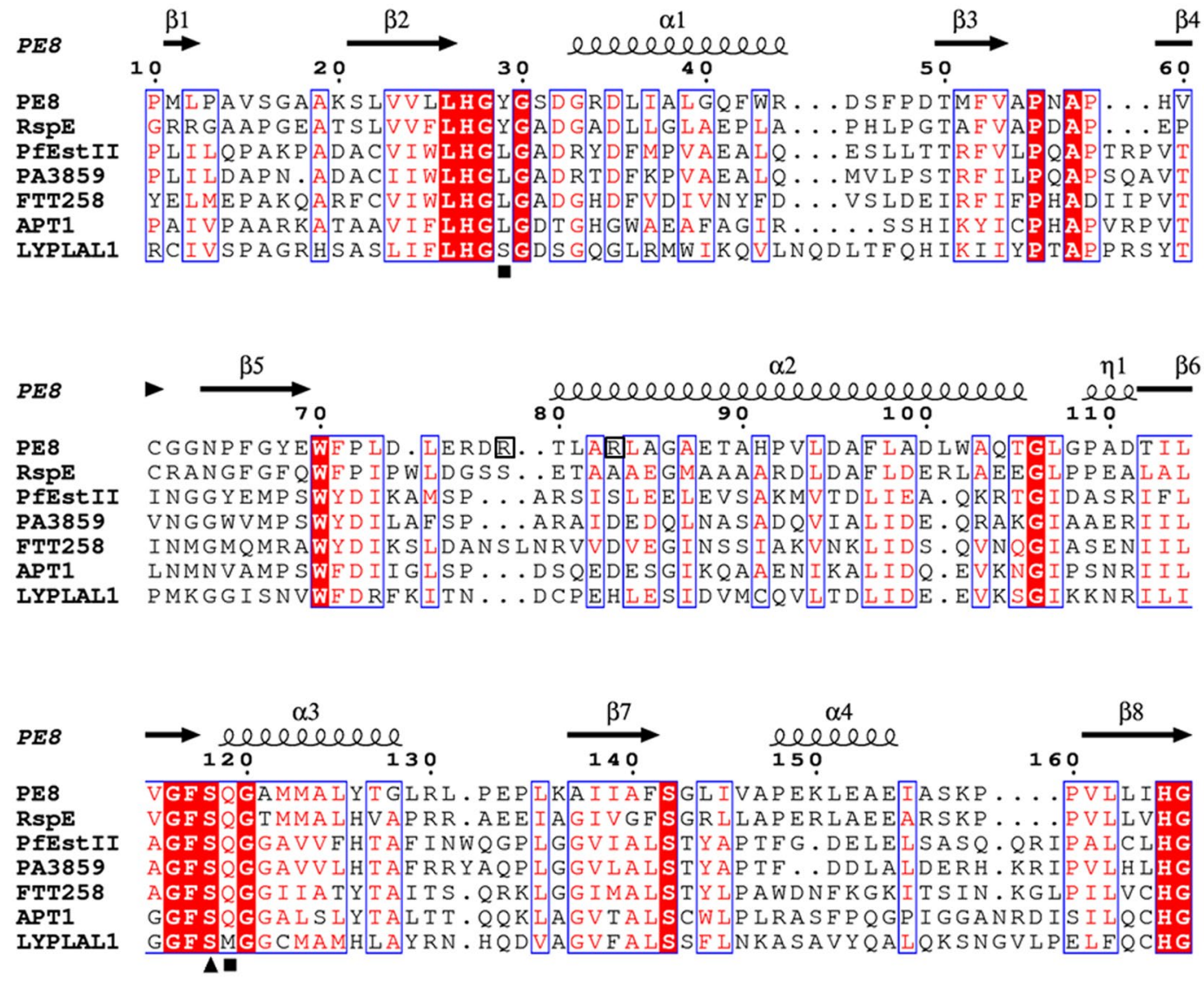

$\alpha 5$

PE8

PE8

RspE

PfEstII

PA3859

FTT258

APT1

LYPLAL1

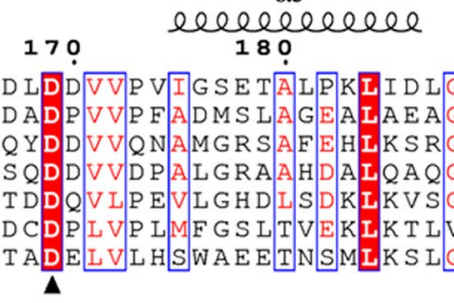

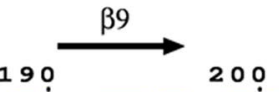

200

\section{. ID AR L HISQGSGH}

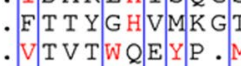

.VIEVGWH $\mathrm{D}$ Y P

F ANEYKHYVGMOHSVCMEEIKDISNF I AKTF

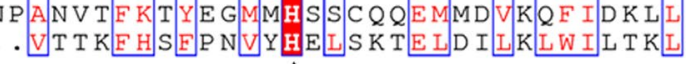

Figure 2. Amino acid sequence alignment of PE8 (PDB: 5DWD) with homologs from the LPCE family. RspE, from R. sphaeroides (PDB: 4FHZ); PfEstII, esterase II from P. fluorescens (PDB: 1AUO); PA3859, from P. aeruginosa (PDB: 3CN9); FTT258, from F. tularensis (PDB: 4F21); APT1, from human (PDB: 1FJ2); LYPLAL1, from human (PDB: 3U0V). Identical and similar residues among groups are shown in white font on a red background and in red font on a white background, respectively. Triangles represent the locations of the catalytic active sites (serine (S), aspartate (D) and histidine $(\mathrm{H})$ ) and squares represent the residues located on the oxyanion hole (tyrosine $(\mathrm{Y}) / \operatorname{tryptophan}(\mathrm{W}) /$ valine $(\mathrm{V}) /$ leucine $(\mathrm{L}) /$ serine $(\mathrm{S})$ and glutamine $(\mathrm{Q}) /$ methionine $(\mathrm{M})$ ). Black boxes represent the locations of residues Arg79 and Arg83 of PE8. The secondary structural elements $\alpha$-helices, $3_{10}$-helices and $\beta$-strands of PE8 are denoted by $\alpha, \eta$ and $\beta$, respectively, with symbols above the sequences.

A new substrate-binding pattern within the LPCE family. PE8 exhibited maximum activity toward the substrate $p$-NP acetate ${ }^{18}$. A docking study of $p$-NP acetate was performed to explore the interaction between PE8 and the substrate. The substrate successfully docked into the active site of PE8 (Fig. 5A), where a PEG MME 550 molecule was detected in the crystal structure (Fig. 5B). In the enzyme-substrate complex docking model, the alcohol part of the substrate occupied the hydrophobic substrate-binding pocket, which was formed by hydrophobic residues Leu73, Met122, Val171 and Val172 (Fig. 5A), similar to the previously suggested mechanism in the LPCE family ${ }^{11}$. The distance between the residues and the nearest carbon atom of the alcohol part of the substrate was 3.3-4.1 A. More importantly, we found that the two nitro-O atoms of $p$-NP acetate might form one and two hydrogen bonds with the side chain $\mathrm{N}$ atoms of Arg79 and Arg83, respectively (Fig. 5A). To the best of our knowledge, this hydrogen bond between the alcohol part of the substrate and the binding site of the esterase (i.e., not the catalytic site) has not been reported before ${ }^{11-14}$. 

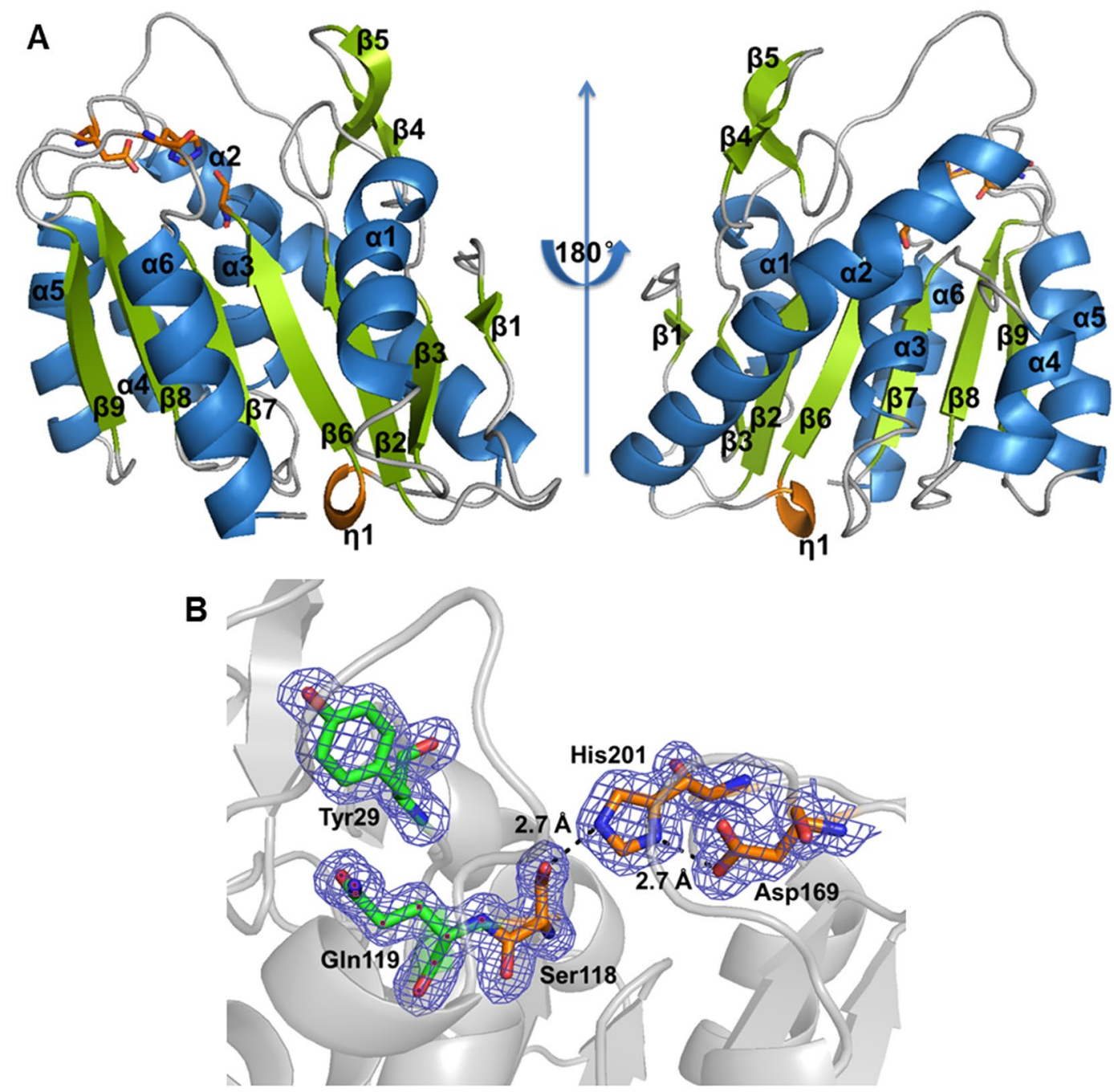

Figure 3. Schematic representation of PE8 structure. (A) Cartoon representation of PE8. The $\alpha$-helices, $\beta$-strands, and $3_{10}$-helices are shown in blue, green, and brown, respectively. The catalytic triad residues are indicated as stick models in orange. (B) Visualization of the PE8 active site. The residues of the catalytic triad and oxyanion hole are shown as stick models in orange and green, respectively. Residue His201 forms hydrogen bonds with Ser118 and Asp169. The electronic map is contoured to $1.0 \sigma$ at the $2 F_{o}-F_{c}$ map.

\begin{tabular}{|c|c|c|c|c|c|c|}
\hline Esterase & PDB ID & Z score & $\operatorname{RSMD}(\AA)$ & NALI $^{\mathrm{a}}$ & NRES $^{b}$ & Identity (\%) \\
\hline R. sphaeroides $\mathrm{RspE}$ & $4 \mathrm{FHZ}$ & 33.9 & 1.7 & 212 & 220 & 46 \\
\hline P. fluorescens esterase II & $1 \mathrm{AUO}$ & 28.0 & 2.1 & 208 & 218 & 24 \\
\hline P. aeruginosa PA3859 & $3 \mathrm{CN} 9$ & 27.8 & 2.4 & 207 & 214 & 26 \\
\hline Human APT1 & $1 \mathrm{FJ} 2$ & 27.0 & 2.3 & 208 & 229 & 23 \\
\hline Human LYPLAL1 & $3 \mathrm{U} 0 \mathrm{~V}$ & 25.4 & 2.4 & 203 & 222 & 22 \\
\hline F. tularensis FTT258 & $4 \mathrm{~F} 21$ & 24.6 & 2.1 & 197 & 220 & 22 \\
\hline
\end{tabular}

Table 2. Structural homologs of PE8 as revealed by DALI ${ }^{22}$. ${ }^{\mathrm{a} N A L I}$ : number of aligned residues; ${ }^{\mathrm{b}} \mathrm{NRES}$ : total number of residues.

In addition, two different configurations of the side chain of Arg79 were observed in chain A and chain B of PE8 (Fig. 5B and C). The configuration of Arg79 in chain B was proposed as the substrate-binding configuration because it was occupied by the PEG molecule, whereas that in chain A might correspond to the releasing state of the substrate-binding site. Thus, the substrate might be bound and subsequently released by the configuration switching of Arg79. Arg79 is located on the $\beta 3$ loop of the structure of PE8. It forms a closed wall between the long winding $\beta 3$ loop and the loop between strand $\beta 8$ and helix $\alpha 5$, in which the Asp169, Val171 and Val172 are located (Figs 2 and 6A). This wall makes the alcohol binding pocket of PE8 a relatively closed cavity and forms stronger interactions with the alcohol group of the ester substrate. However, because of the high sequence 

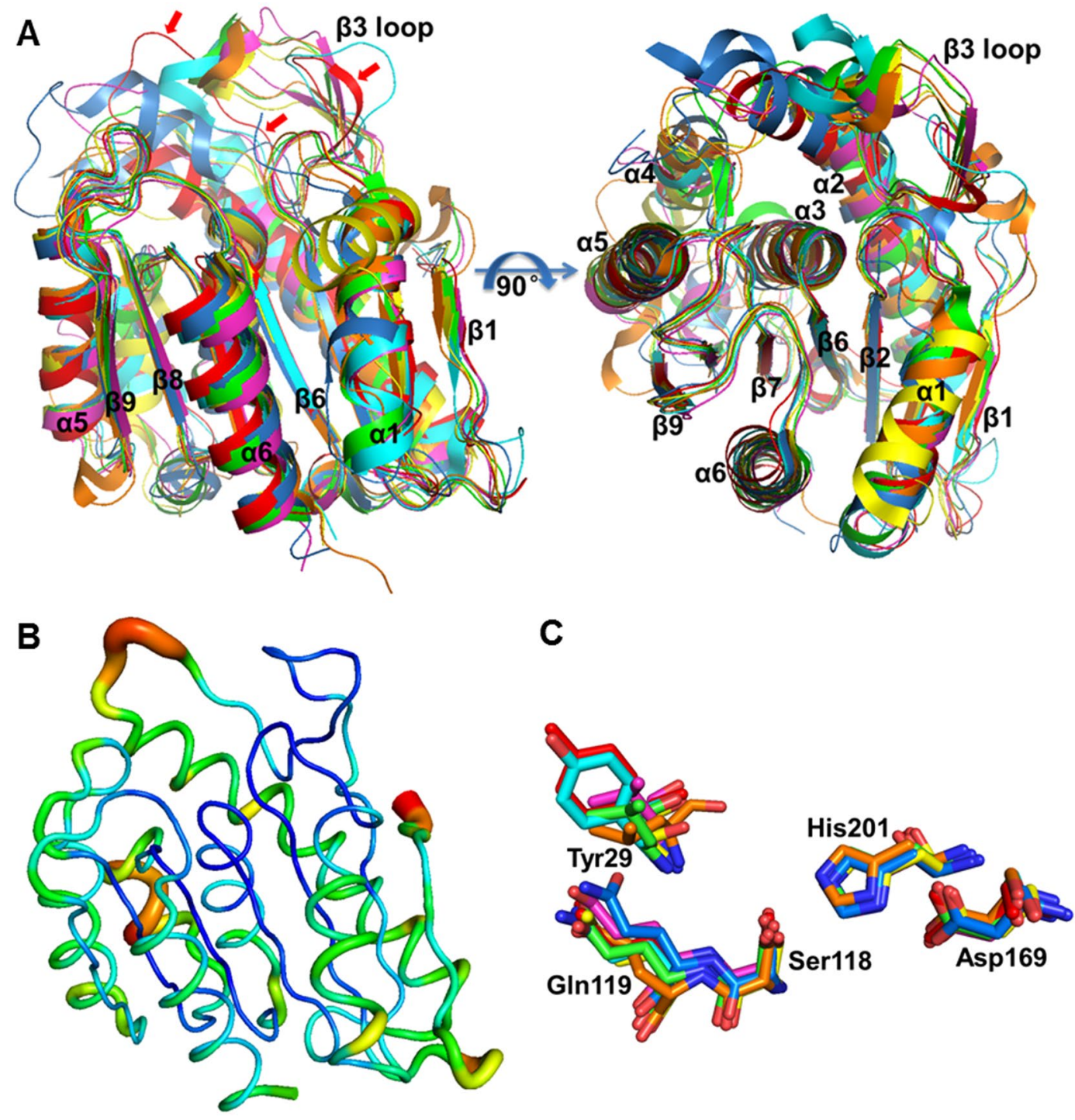

Figure 4. A structural comparison of PE8 with other homologous esterases. (A) The structural superposition of PE8 (red, PDB: 5DWD), R. sphaeroides RspE (cyan, PDB: 4FHZ), P. fluorescens esterase II (magentas, PDB: 1AUO), P. aeruginosa PA3859 (green, PDB: 3CN9), human APT1 (orange, PDB: 1FJ2), LYPLAL1 (yellow, PDB: 3U0V) and F. tularensis FTT258 (blue, PDB: 4F21), which was performed in PyMOL using C $\alpha$ atoms with default parameters. The $\beta$-strands, $\alpha$-helices and winding $\beta 3$ loops are labeled. Red arrows indicate the $\beta 3$ loop of PE8. (B) The B-factor distribution of PE8. Wider and redder tubing corresponds to higher B-factors. (C) The comparison of the catalytic triads and oxyanion holes among the seven homologous proteins. The five residues are presented as stick models. The colors have the same meaning as in (A).

variability and structural flexibility of loop $\beta 3$ (Figs 2 and 4), no similar structure was found in other homologs of the LPCE family. For example, $R$. sphaeroides RspE has the highest sequence and structural similarity with PE8; however, the substrate-binding pocket of $R$. sphaeroides RspE is an open, longer cleft (Fig. 6B), similar to other members of the LPCE family ${ }^{11,13}$. Hence, Arg79 may play an important role in substrate binding and the shape of the binding pocket, thereby conferring substrate specificity to PE8.

To investigate the relationship between the supposed substrate-binding-related residues and the hydrolysis activity of PE8, mutants L73A, R79A, R83A, M122A, V171A and V172A were constructed by site-directed mutagenesis, and their catalytic activities against $p$-NP esters and kinetic parameters for the hydrolysis of $p$-NP acetate were determined (Fig. $1 \mathrm{C}$ and Table 3 ). The $\mathrm{Km}, k \mathrm{cat}$ and $k \mathrm{cat} / \mathrm{Km}$ of wild-type PE8 were $0.66 \pm 0.049 \mathrm{mM}$, $30 \pm 0.68 \mathrm{~s}^{-1}$ and $45 \mathrm{mM}^{-1} \mathrm{~s}^{-1}$, respectively. The catalytic activities and $K \mathrm{~m}$ and $k$ cat values of L73A, R83A and V172A were similar to those of wild-type PE8, indicating that these mutations had little effect on the catalytic activity and substrate affinity of PE8 using $p$-NP esters. A slight increase in the Km value and decrease in the $k$ cat value of mutant V171A were observed $\left(0.89 \pm 0.070 \mathrm{mM}\right.$ and $19 \pm 0.42 \mathrm{~s}^{-1}$, respectively), suggesting that the ability to bind the substrate was weakened and that the turnover rate of the enzyme-substrate complex to the product and enzyme was decreased. Thus, the catalytic efficiency against $p$-NP acetate of mutant V171A was reduced by nearly half, as indicated by a $k \mathrm{cat} / K \mathrm{~m}$ value of $21 \mathrm{mM}^{-1} \mathrm{~s}^{-1}$. The $k$ cat and $k \mathrm{cat} / K \mathrm{~m}$ values of mutant 


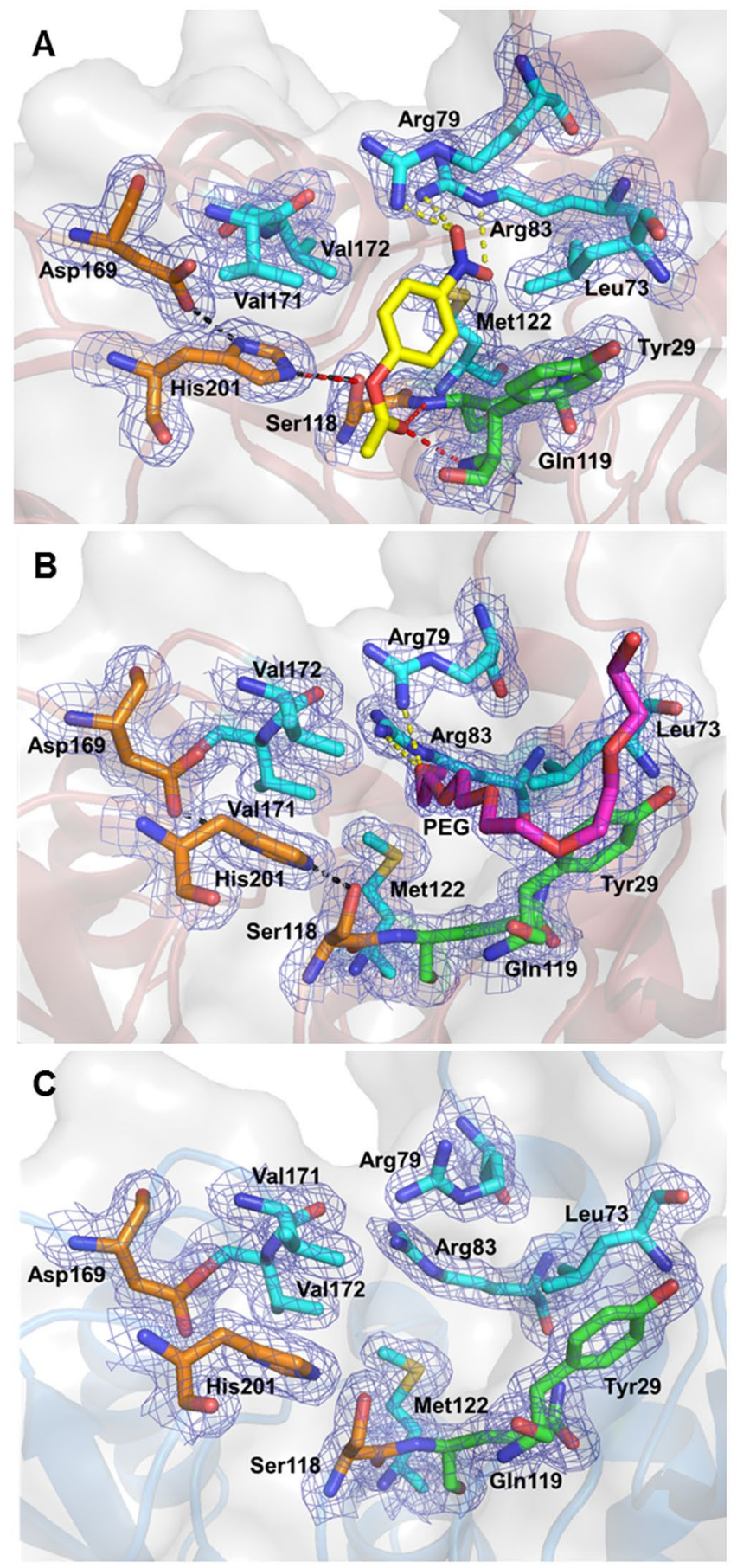

Figure 5. Visualization of the substrate-binding site of PE8. (A) The structural model of PE8 and its complex with substrate $p$-NP acetate (yellow sticks). (B) The PEG MME 550 molecule in the substrate-binding site of PE8 chain B. (C) The substrate-binding site of PE8 chain A (cartoon in blue), showing the different configuration of Arg79 relative to chain A. All the electronic maps are contoured to $1.0 \sigma$ at the $2 F_{o}-F_{c}$ map. The residues Leu73, Arg79, Arg83, Met122, Val171 and Val172, the catalytic triad and the oxyanion hole of PE8 are shown as stick models. Residue His201 forms hydrogen bonds with Ser118 and Asp169 (black dotted lines). The substrate molecule or PEG MME 550 forms hydrogen bonds with His201, Tyr29 and Gln119 (red dotted lines) and Arg79 and Arg83 (yellow dotted lines).

R79A were approximately 2-fold higher than those of wild-type PE8. Considering the structure of Arg79 shown above, replacing arginine with alanine probably removed the barrier and expanded the substrate-binding pocket, thereby accelerating substrate access and exit. Interestingly, mutant M122A showed a large decrease in its $\mathrm{Km}$ value $(0.075 \pm 0.0069 \mathrm{mM})$ toward $p$-NP acetate, resulting in a 10 -fold improvement of $k \mathrm{cat} / \mathrm{Km}\left(553 \mathrm{mM}^{-1} \mathrm{~s}^{-1}\right)$. In addition, the catalytic activities of mutant M122A against $p$-NP butyrate, $p$-NP hexanoate and $p$-NP octanoate also increased relative to those of wild-type PE8 (Fig. 1C). The substitution of methionine by alanine might cause 

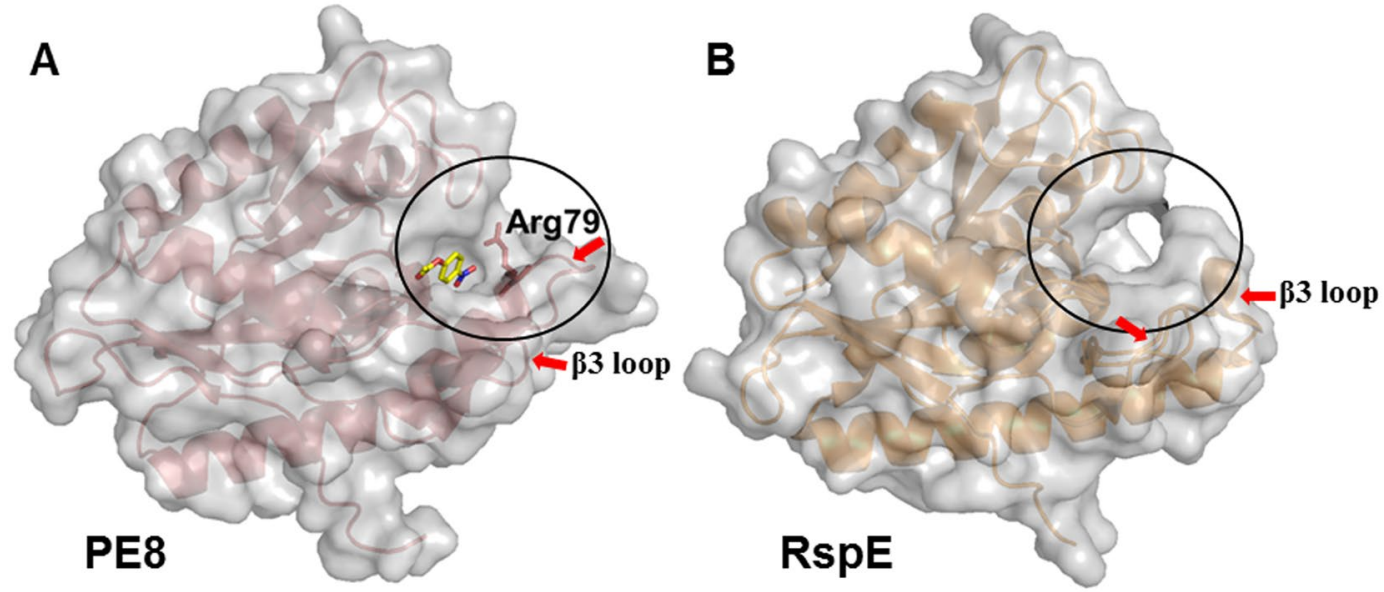

Figure 6. Molecular surface representation (light grey) of PE8 and R. sphaeroides RspE (PDB 4FHZ). (A) p-NP acetate docks into the substrate-binding pocket of $\mathrm{PE} 8$ chain $\mathrm{B}$, which is shown as a cartoon representation in red (Arg79 is shown as a stick). (B) RspE is shown as a cartoon representation in orange. The relatively closed cavity of the substrate-binding pocket caused by Arg79 in PE8 and the open cleft in RspE are indicated with black circles. Red arrows indicate the $\beta 3$ loops.

\begin{tabular}{|l|l|l|l|l|}
\hline Enzyme & $V \mathbf{m a x}(\boldsymbol{\mu M} / \mathbf{m i n} / \mathbf{m g})$ & $\mathbf{K m}(\mathbf{m M})$ & $k$ cat $\left(\mathbf{s}^{-1}\right)$ & $k$ cat $/ \mathbf{K m}\left(\mathbf{m M}^{-1} \mathbf{s}^{-\mathbf{1}}\right)^{\mathbf{a}}$ \\
\hline PE8 & $71 \pm 1.6$ & $0.66 \pm 0.049$ & $30 \pm 0.68$ & $45(100 \%)$ \\
\hline L73A & $55 \pm 0.64$ & $0.53 \pm 0.026$ & $23 \pm 0.27$ & $43(96 \%)$ \\
\hline R79A & $132 \pm 3.4$ & $0.60 \pm 0.062$ & $55 \pm 1.7$ & $92(204 \%)$ \\
\hline R83A & $97 \pm 1.0$ & $0.60 \pm 0.023$ & $41 \pm 0.42$ & $68(151 \%)$ \\
\hline M122A & $96 \pm 1.6$ & $0.075 \pm 0.0069$ & $40 \pm 0.68$ & $533(1184 \%)$ \\
\hline V171A & $36 \pm 1.8$ & $0.89 \pm 0.070$ & $19 \pm 0.42$ & $21(47 \%)$ \\
\hline V172A & $82 \pm 1.5$ & $0.50 \pm 0.035$ & $35 \pm 0.63$ & $70(156 \%)$ \\
\hline S118A & $12 \pm 0.32$ & $0.54 \pm 0.046$ & $5.1 \pm 0.14$ & $9.4(21 \%)$ \\
\hline
\end{tabular}

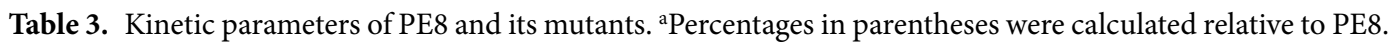
All catalytic reactions were performed in triplicate in $100 \mathrm{mM}$ Tris- $\mathrm{HCl}$ buffer $(\mathrm{pH} 7.5)$ buffer at $30^{\circ} \mathrm{C}$ using $p$ nitrophenyl acetate as the substrate at concentrations of $0.05-4 \mathrm{mM}$.

less steric repulsion of the substrates and remove the barrier to substrate access. Hence, further engineering in positions Arg79, Met122 or Val171 may provide high activity, affinity or selectivity mutants to specific substrates to facilitate the development of structure-based therapeutics and other biocatalytic applications.

\section{Conclusion}

In this study, a new esterase structure in the LPCE family is presented. The structural information of P. halotolerans PE8 expands our knowledge of the catalytic mechanisms of the LPCE family and provides new insight into the substrate-binding pattern in this family. The results establish a novel approach for developing specific inhibitors of its homologs, which could be used for mechanistic research and targeted therapy. In addition, the results of this paper may help broaden the applications of the LPCE family members as biocatalysts in industry.

\section{Materials and Methods}

Sequence analysis. The PE8 coding gene was identified and cloned from the genome of P. halotolerans $\mathrm{B} 2^{\mathrm{T} 16,18}$. Amino acid sequence analysis was conducted by BLASTp against the PDB from the National Center for Biotechnology Information (NCBI). Multiple sequence alignment of homologs belonging to the LPCE family was performed by ClustalX v. $2^{26}$. Secondary structure assignment was determined by DSSP v. $2.0^{27}$ and PROMOTIF $^{28}$. The alignment result with the secondary structure was visualized using ESPript 3.0 ${ }^{29}$.

Mutation, protein expression and purification. Point mutants were generated by site-directed mutagenesis using wild-type plasmid as a template for the polymerase chain reaction (PCR). A 15-cycle reaction was performed with the following steps: $98^{\circ} \mathrm{C}$ for $10 \mathrm{sec}, 55^{\circ} \mathrm{C}$ for $30 \mathrm{sec}$, and $72^{\circ} \mathrm{C}$ for $3 \mathrm{~min}$ per cycle with PrimeSTAR HS DNA polymerase (Takara, Dalian, Liaoning, China). After digestion with enzyme DpnI (New England Biolabs, Beverly, MA, USA), the PCR products were transformed into Escherichia coli $\mathrm{DH} 5 \alpha$ cells. The positive constructs were determined by DNA sequencing. The wild-type and mutant forms of PE8 were cloned into the expression vector pET28b (Novagen, Madison, WI, USA) and were expressed in E. coli Rosetta (DE3) cells induced by $0.5 \mathrm{mM}$ isopropyl- $\beta$-D-thiogalactopyranoside (IPTG) for 16 hours at $20^{\circ} \mathrm{C}$, as described previously ${ }^{18,30}$. Cells were harvested and disrupted by a sonicator or high-pressure homogenizer. The lysates were sequentially purified by 
Ni-NTA affinity and size-exclusion chromatographys (SEC). The Superdex-200 column was calibrated with protein size markers: thyroglobulin $(670 \mathrm{kDa})$, gamma globulin $(158 \mathrm{kDa})$, ovalbumin $(44 \mathrm{kDa})$, myoglobin $(17 \mathrm{kDa})$ and vitamin B12 (1.35 kDa). The protein concentration was determined by the Bradford method.

MALS analysis. MALS analysis was performed to estimate the MW of PE8 in the National Center for Protein Science Shanghai (NCPSS). First, $20 \mu \mathrm{l}$ of $1.5 \mathrm{mg} / \mathrm{ml}$ purified PE8 protein was subjected to SEC-MALS using a WTC-030S5 size-exclusion column (Wyatt, Santa Barbara, CA, USA) with elution buffer (20 mM Tris- HCl, $\mathrm{pH}$ 7.4, $100 \mathrm{mM} \mathrm{NaCl}$ ) and passed in tandem through a Wyatt DAWN HELEOS II light scattering instrument (Wyatt) and an Optilab rEX refractometer (Wyatt). Data collection and analysis were performed with Astra 6 software (Wyatt).

Biochemical characterization of PE8 and its mutants. The standard reaction was carried out with the appropriate amount of purified PE8 or its mutants in $1 \mathrm{ml}$ mixtures containing $100 \mathrm{mM}$ Tris- $\mathrm{HCl}(\mathrm{pH} 7.5)$ buffer and $1 \mathrm{mM} p$-NP acetate (Sigma-Aldrich, Milwaukee, WI, USA, dissolved in acetonitrile) ${ }^{18}$. The activities were determined at $30^{\circ} \mathrm{C}$ and $405 \mathrm{~nm}$ using a DU800 UV/Visible spectrophotometer (Beckman, Houston, TX, USA). All experiments were performed in triplicate and corrected for substrate autohydrolysis. Substrate specificity assays were performed with $p$-NP acetate, $p$-NP butyrate (Sigma-Aldrich), $p$-NP hexanoate (TCI, Tokyo, Japan) and $p$-NP octanoate (Sigma-Aldrich).

The kinetic parameters were obtained using $p$-NP acetate as a substrate at different concentrations $(0.05$ to $4 \mathrm{mM}$ ). The kinetic parameters were calculated by analyzing the slopes of the Michaelis-Menten equation using GraphPad Software (GraphPad Inc., USA).

Crystallization, data collection, and structure determination. Crystals of PE8 protein were obtained using the "hanging drop" method by mixing $1 \mu \mathrm{l}$ of $20 \mathrm{mg} / \mathrm{ml}$ protein with $1 \mu \mathrm{l}$ of reservoir solution at $20^{\circ} \mathrm{C}$. The reservoir buffer contained $0.05 \mathrm{M} \mathrm{CaCl}_{2}, 0.1 \mathrm{M}$ Bis-Tris, and 25\% (v/v) PEG MME 550 (pH 6.5). The X-ray diffraction datasets were integrated, scaled and merged using the HKL3000 program ${ }^{31}$. Phases were obtained by molecular replacement using Phaser ${ }^{32}$ with the PDB coordinates 4 FHZ (R. sphaeroides RspE $)^{14}$ as the initial model. The refinement was conducted by Refmac $5^{33}$ in the CCP4 software suite ${ }^{34}$ and Phenix ${ }^{35}$. The model was built manually by $\operatorname{Coot}^{36}$. A structural similarity search was performed with the DALI server ${ }^{24}$. Docking studies were performed with AutoDockTools program ${ }^{37}$. The ligands for docking were edited by Avogadro software ${ }^{38}$, and the topologies of the ligands were generated using the PRODRG server ${ }^{39}$. The successful docking conformation should be satisfied the following criteria: the distance between the OG atom of serine and the carbonyl carbon atom of the substrate was about $2 \AA$; the catalytic hydrogen bonds were formed, including that between $\mathrm{H} \delta$ of the catalytic histidine and the ester oxygen of the substrate, as well as those between the carbonyl oxygen of the substrate and the nitrogen atoms of oxyanion hole residues (Tyr29 and Gln119) ${ }^{40,41}$. All the structures were drawn using PyMOL software (http://pymol.sourceforge.net).

The structure of PE8 was deposited in PDB with accession number 5DWD.

\section{References}

1. Arpigny, J. L. \& Jaeger, K. E. Bacterial lipolytic enzymes: classification and properties. The Biochemical Journal 343, 177-183 (1999).

2. Hosokawa, M. Structure and catalytic properties of carboxylesterase isozymes involved in metabolic activation of prodrugs. Molecules 13, 412-431 (2008).

3. Panda, T. \& Gowrishankar, B. Production and applications of esterases. Applied Microbiology and Biotechnology 67, 160-169 (2005).

4. Lenfant, N. et al. ESTHER, the database of the $\alpha / \beta$-hydrolase fold superfamily of proteins: tools to explore diversity of functions. Nucleic Acids Research, D423-D429 (2012).

5. Dekker, F. J. et al. Small-molecule inhibition of APT1 affects Ras localization and signaling. Nature Chemical Biology 6, 449-456 (2010).

6. Rocks, O. et al. An acylation cycle regulates localization and activity of palmitoylated Ras isoforms. Science 307, 1746-1752 (2005).

7. Bürger, M. et al. Crystal structure of the predicted phospholipase LYPLAL1 reveals unexpected functional plasticity despite close relationship to acyl protein thioesterases. Journal of Lipid Research 53, 43-50 (2012).

8. Steinberg, G. R., Kemp, B. E. \& Watt, M. J. Adipocyte triglyceride lipase expression in human obesity. American Journal of PhysiologyEndocrinology and Metabolism 293, E958-E964 (2007).

9. Larsson, P. et al. The complete genome sequence of Francisella tularensis, the causative agent of tularemia. Nature Genetics 37, 153-159 (2005).

10. McLendon, M. K., Apicella, M. A. \& Allen, L.-A. H. Francisella tularensis: taxonomy, genetics, and immunopathogenesis of a potential agent of biowarfare. Annual Review of Microbiology 60, 167 (2006).

11. Filippova, E. V. et al. Large scale structural rearrangement of a serine hydrolase from Francisella tularensis facilitates catalysis. Journal of Biological Chemistry 288, 10522-10535 (2013).

12. Kim, K. K. et al. Crystal structure of carboxylesterase from Pseudomonas fluorescens, an alpha/beta hydrolase with broad substrate specificity. Structure 5, 1571-1584 (1997).

13. Pesaresi, A. \& Lamba, D. Insights into the fatty acid chain length specificity of the carboxylesterase PA3859 from Pseudomonas aeruginosa: A combined structural, biochemical and computational study. Biochimie 92, 1787-1792 (2010).

14. Ma, J. et al. Enhanced enantioselectivity of a carboxyl esterase from Rhodobacter sphaeroides by directed evolution. Applied Microbiology and Biotechnology 97, 4897-4906 (2013).

15. Devedjiev, Y., Dauter, Z., Kuznetsov, S. R., Jones, T. L. Z. \& Derewenda, Z. S. Crystal structure of the human acyl protein thioesterase I from a single X-ray data set to $1.5 \AA$. Structure 8, 1137-1146 (2000).

16. Huo, Y.-Y. et al. Complete genome sequence of Pelagibacterium halotolerans B2T. Journal of Bacteriology 194, 197-198 (2012).

17. Xu, X.-W. et al. Pelagibacterium halotolerans gen. nov., sp. nov. and Pelagibacterium luteolum sp. nov., novel members of the family Hyphomicrobiaceae. International Journal of Systematic and Evolutionary Microbiology 61, 1817-1822 (2011).

18. Wei, X. et al. Cloning, expression and characterization of a new enantioselective esterase from a marine bacterium. Pelagibacterium halotolerans B2T. Journal of Molecular Catalysis B: Enzymatic 97, 270-277 (2013).

19. Liu, W., Hu, Y., Jiang, L., Zou, B. \& Huang, H. Synthesis of methyl (R)-3-(4-fluorophenyl)glutarate via enzymatic desymmetrization of a prochiral diester. Process Biochemistry 47, 1037-1041 (2012).

20. Yu, M. S., Lantos, I., Peng, Z.-Q., Yu, J. \& Cacchio, T. Asymmetric synthesis of (-)-paroxetine using PLE hydrolysis. Tetrahedron Letters 41, 5647-5651 (2000). 
21. Holmquist, M. Alpha/Beta-hydrolase fold enzymes: structures, functions and mechanisms. Current protein \& peptide science $\mathbf{1}$, 209-235 (2000)

22. Li, J. et al. Crystal structure of human guanosine monophosphate reductase 2 (GMPR2) in complex with GMP. Journal of molecular biology 355, 980-988 (2006)

23. Lee, J.-H. et al. Crystal structure and versatile functional roles of the COP9 signalosome subunit 1. Proceedings of the National Academy of Sciences 110, 11845-11850 (2013).

24. Holm, L. \& Rosenström, P. Dali server: conservation mapping in 3D. Nucleic Acids Research 38, W545-W549 (2010).

25. van Tilbeurgh, H. et al. Interfacial activation of the lipase-procolipase complex by mixed micelles revealed by X-ray crystallography. Nature 362, 814-820 (1993).

26. Larkin, M. A. et al. Clustal W and Clustal X version 2.0. Bioinformatics (Oxford, England) 23, 2947-2948 (2007).

27. Kabsch, W. \& Sander, C. Dictionary of protein secondary structure: pattern recognition of hydrogen-bonded and geometrical features. Biopolymers 22, 2577-2637 (1983).

28. Hutchinson, E. G. \& Thornton, J. M. PROMOTIF-a program to identify and analyze structural motifs in proteins. Protein Science 5, 212-220 (1996).

29. Robert, X. \& Gouet, P. Deciphering key features in protein structures with the new ENDscript server. Nucleic Acids Research 42, W320-W324 (2014).

30. Huang, J. et al. Structural insights of a hormone sensitive lipase homologue Est22. Scientific Reports 6 (2016).

31. Minor, W., Cymborowski, M., Otwinowski, Z. \& Chruszcz, M. HKL-3000: the integration of data reduction and structure solution-from diffraction images to an initial model in minutes. Acta Crystallographica Section D: Biological Crystallography 62, 859-866 (2006)

32. McCoy, A. J. et al. Phaser crystallographic software. Journal of Applied Crystallography 40, 658-674 (2007).

33. Murshudov, G. N., Vagin, A. A. \& Dodson, E. J. Refinement of macromolecular structures by the maximum-likelihood method. Acta Crystallographica Section D: Biological Crystallography 53, 240-255 (1997).

34. Winn, M. D. et al. Overview of the CCP4 suite and current developments. Acta Crystallographica Section D: Biological Crystallography 67, 235-242 (2011).

35. Adams, P. D. et al. PHENIX: a comprehensive Python-based system for macromolecular structure solution. Acta Crystallographica Section D: Biological Crystallography 66, 213-221 (2010).

36. Emsley, P. \& Cowtan, K. Coot: model-building tools for molecular graphics. Acta Crystallographica Section D: Biological Crystallography 60, 2126-2132 (2004).

37. Morris, G. M. et al. AutoDock4 and AutoDockTools4: Automated docking with selective receptor flexibility. Journal of Computational Chemistry 30, 2785-2791 (2009).

38. Hanwell, M. D. et al. Avogadro: An advanced semantic chemical editor, visualization, and analysis platform. Journal of Cheminformatics 4, 17 (2012).

39. SchuEttelkopf, A. W. \& Van Aalten, D. M. F. PRODRG: a tool for high-throughput crystallography of protein-ligand complexes. Acta Crystallographica Section D: Biological Crystallography 60, 1355-1363 (2004).

40. Gu, J., Liu, J. \& Yu, H. Quantitative prediction of enantioselectivity of Candida antarctica lipase B by combining docking simulations and quantitative structure-activity relationship (QSAR) analysis. Journal of Molecular Catalysis B Enzymatic 72, 238-247 (2011).

41. Guo, F., Franzen, S., Ye, L., Gu, J. \& Yu, H. Controlling enantioselectivity of esterase in asymmetric hydrolysis of aryl prochiral diesters by introducing aromatic interactions. Biotechnology and bioengineering 111, 1729-1739 (2014).

\section{Acknowledgements}

We thank the staff from the BL17U1 beamline at Shanghai Synchrotron Radiation Facility and BL18U1/ BL19U1 beamlines of the National Center for Protein Sciences Shanghai (NCPSS) for assistance during data collection. This work was supported by grants from the National Key Research and Development Project of China (2016YFA0500600, 2015CB943300), the National Natural Science Foundation of China $(41506183,31470724)$, Open Research Program of the Key Laboratory of Microbial Resources Collection and Preservation, Ministry of Agriculture, the Top-Notch Young Talents Program of China, and Laboratory of Marine Ecosystem and Biogeochemistry (LMEB), SOA Open Research Funding (LMEB201503).

\section{Author Contributions}

X.-W.X. and J.L. conceived and designed the study; Y.-Y.H., J.H. and R.J. performed crystallization, data collection and structural refinement; S.L., Z.R., Z.W., Z.L. and H.-L.C. performed biochemical assays. Y.-Y.H., J.L. and X.W.X. wrote the manuscript. All authors reviewed the manuscript.

\section{Additional Information}

Supplementary information accompanies this paper at doi:10.1038/s41598-017-04550-7

Competing Interests: The authors declare that they have no competing interests.

Accession codes: The coordinates and structural factors of PE8 have been deposited in the Protein Data Bank with accession codes 5DWD.

Publisher's note: Springer Nature remains neutral with regard to jurisdictional claims in published maps and institutional affiliations.

Open Access This article is licensed under a Creative Commons Attribution 4.0 International

License, which permits use, sharing, adaptation, distribution and reproduction in any medium or format, as long as you give appropriate credit to the original author(s) and the source, provide a link to the Creative Commons license, and indicate if changes were made. The images or other third party material in this article are included in the article's Creative Commons license, unless indicated otherwise in a credit line to the material. If material is not included in the article's Creative Commons license and your intended use is not permitted by statutory regulation or exceeds the permitted use, you will need to obtain permission directly from the copyright holder. To view a copy of this license, visit http://creativecommons.org/licenses/by/4.0/.

(C) The Author(s) 2017 